\title{
Regression Model using Instance based Learning Streams
}

\author{
P. K. Srimani ${ }^{1}$ and Malini M. Patil ${ }^{2 *}$ \\ 'R\&D Division, Bangalore University, Bangalore, Karnataka, India; profsrimanipk@gmail.com \\ 2Department of ISE, J.S.S. Academy of Technical Education, Bangalore, Karnataka, India; patilmalini31@yahoo.com
}

\begin{abstract}
Data mining is concerned with the analysis of data for finding patterns and regularities in the data sets. Statistics is a mathematical science concerned with the collection, analysis, interpretation or explanation, and presentation of data. Statistics plays a very important role in the process of data mining analysis and equally visualization of data plays a very important role in decision making process. Instance Based Learning Streams is an instance-based learning algorithm used to perform regression analysis on data streams. The algorithm is able to handle large data streams with less memory and computational power. The paper aims at the implementation of Instance Based Learning Streams as an extension to the massive online analysis framework for data stream mining to develop a regression model. The study reveals that the regression analysis could be performed not only on small data sets but also on data streams as in the present case but the method of analysis will be different in the two cases. In the case of small data set the regression models are linear, multiple and polynomial, while in the case of data streams the entire analysis is performed under the massive online analysis framework by taking the two evaluation parameters basic regression performance evaluator and windows regression performance evaluator. This finding is first of its kind in literature.
\end{abstract}

Keywords: Data Streams, IBLStreams, Instance, Prediction, Massive Online Analysis, Regression

\section{Introduction}

Advancement of technology has resulted in large storage of data. These large masses of data consist of some hidden information of strategic importance, which can be used for future analysis with effective decision making. The two important types of data analysis methods are classification and prediction. In the former case a model is constructed (classifier) to predict the categorical labels, in the latter case a model is constructed (predictor) to predict the continuous variables. Regression analysis is a statistical method which is widely used for prediction and forecasting. It is also considered as the part of machine learning process. It plays a very important role in prediction. The authors ${ }^{1}$ have explained thoroughly about regression analysis. It can be used to model the relationship between one or more independent variables and a dependent variable.
Different techniques are developed to carry out regression analysis, namely, Linear regression, multiple regression, polynomial regression and ordinary least squares regression. These methods can be parametric or non parametric in nature. Many tools are also developed to carry out regression analysis viz. miniTab, Gnumeric, PASW. Regression analysis can be performed very efficiently using MS-Excel also.

Rapid advancement of the technology resulted in the storage of digital data has also increased very rapidly. The continuous arrival of data is referred to as data stream. Network monitoring data, sensor data, web clicks, usage of credit cards weather forecasting data are few examples of data streams. The data streams are massive in nature and they arrive at a very high speed. Data mining techniques are not suitable for mining data streams and the data streams must be processed under very strict constraints of space and time. Gaber et al. ${ }^{2}$, Gaber and

${ }^{*}$ Author for correspondence 
Gama $^{3}$ and Ikonomovska ${ }^{4}$ have explained many aspects of data streams, different characteristics and many other special features of data streams. Data streams can be mined using Massive Online Analysis (MOA) frame work. Basic data mining techniques, classification, clustering and association rule mining can be performed on data streams using MOA framework. The present work mainly aims at regression analysis using IBLS in MOA framework.

The paper is organised as follows: Section 2 mainly discusses on the related work in the area of regression modelling and need and importance of the problem; Section 3 about the IBL stream learning Section 4 discusses about methodology about regression analysis in MOA; Experiments and results are presented in Section 5; Finally, section 6 is about conclusions and future work.

\section{Related Work}

A thorough survey of the literature reveals that very sparse literature is available pertaining to the present work. The work done in this regard is briefed in this section. Most of the related work was found on the analysis and visualization of data. The objective of the analysis and visualization of data is to highlight useful information and support decision making. The authors emphasize the use of statistical approach in Edu_data, generated from technical education system which consists of three stake holders namely Student, Faculty and Management. Authors have exhaustively studied linear ${ }^{5-7}$ regression analysis using PASW-18 statistical tool ${ }^{8}$ on Edu-Data and results were found to be very accurate and it is one of its kind on edu data. Overall the study reveals that the regression model is developed on static data.

As the technology is advanced the data collection is found to be massive in nature and such a data is referred to as data streams ${ }^{9}$ or massive data. Later study reveals that the possibility of regression modeling on massive data, as till now it was restricted only on a small sample of data. The author ${ }^{10}$ proposed a new method for mining large data sets using regression classes. It is defined as subset of the large data set which can be used for regression modeling. A large data set is treated as a mixture of many such regression classes. Fan ${ }^{11}$ consider block weighted least square estimators of the regression coefficients by minimizing the variances of the estimators and prove the asymptotic properties of the resulting estimators. They also indicate that the estimators make better interval estimation in terms of coverage probabilities than the usual least square estimators.

The recent work carried out by Shakeer ${ }^{12}$, presents an approach to learning on data streams called Instance Based Learning Streams (IBLS). They have introduced the main methodological concepts underlying this approach by a mathematical model and implementation under MOA framework using a fuzzy model called as FLEXFIS ${ }^{13}$. MOA frame work was introduced by Bifet et al. ${ }^{14-17}$. Subsequent citations Bifet et al. ${ }^{18}$ elaborates the use of IBLstreams as extension in MOA framework. Therefore the present investigation is aimed at developing a regression model using instance based approach using IBLstreams on massive online analysis. The model uses two different prediction strategies Weighted_Mean (W_MEAN_REG) and Local_Linear_Regression (LOC_ LIN_REG) where one Adaptation_Strategy (adapt_k) and two parameter evaluation methods viz., Basic Regression Parameter Evaluator (BRPE) and Window Regression Parameter Evaluation (WRPE) and lastly the hold out evaluation method in MOA frame work are used. Thus the present work provides an excellent platform for future research.

\section{Instance based Learning Streams (IBLS)}

The term instance based learning is more precily explained by Shakeer ${ }^{12}$. The different types of instance based learning are viz., Memory Based Learning (MBL), Exempler Based Learning(EBL) and case based learning. In case of IBLS as the term suggests special importance is given to concept of an instance. An instance or an exemplar can be thought of as a single excerpt, such as pattern or a problem. Model-based machine learning methods induce a general model from the data and use that model for further reasoning. Using this method IBL algorithms simply store the data itself, defer the processing of the data until a prediction is actually requested. Predictions are then derived by combining the information provided by the stored examples.

\subsection{Mathematical Model for IBL}

Consider the following setting: Let $\mathrm{X}$ denote the instance space, where an instance corresponds to the description $\mathrm{x}$ of an object $\mathrm{X}$ and is equipped with a distance measure $\Delta(\cdot)$, i.e., $\Delta\left(\mathrm{x}, \mathrm{x}^{\prime}\right)$ is the distance between instances 
$\mathrm{x}, \mathrm{x}^{\prime} \varepsilon \mathrm{X}$. $\mathrm{Y}$ is the output space and $(\mathrm{x}, \mathrm{y}) \varepsilon \mathrm{X} \times \mathrm{Y}$ is called a labeled instance, a case, or an example. In classification, $\mathrm{Y}$ is a finite (usually small) set comprised of $\mathrm{m}$ classes $\left\{\lambda_{1}, \ldots, \lambda_{\mathrm{m}}\right\}$, where as $\mathrm{Y}=\mathrm{R}$ in regression. The present learning system is represented in terms of a set $\mathrm{D}$ of examples $\left(\mathrm{x}_{\mathrm{i}}\right.$ , $\left.\mathrm{y}_{\mathrm{i}}\right), 1 \leq \mathrm{i} \leq \mathrm{n}=|\mathrm{D}|$. From a machine learning point of view, $\mathrm{D}$ plays the role of the training set of the learner. More precisely, since not all examples will necessarily be stored by an instance-based learner, $\mathrm{D}$ is only a subset of the data.

\section{Regression Analysis in Massive Online Analysis Frame Work}

The present section emphasizes on the step wise explanation pertaining to the basic configuration method in MOA Framework. Shakeer ${ }^{12}$ explained the different possibilities of selecting the prediction and adaption strategies, different weighting methods and selecting the instance width which are basically used as fixed options for the experimantal set up in MOA. This section provides the detailed explanation of the same. It is basically used as an extension to the framework. It is understood from the technique of regression analysis that, the target attribute is numerical and loss is typically measured in terms of the absolute or squared difference between the predicted output and the true output. Similarly the MOA configuration steps are also based on the same strategy.

The prediction problems can be solved in two ways.

- Firstly, the target value can be estimated by the weighted mean of the target values of the $\mathrm{k}$ neighbor instances. This prediction is obtained by selecting the option 'W_Mean_Reg', in the MOA frame work, which sets the Prediction_Strategy parameter to Weighted_Mean_Regression.

- Secondly, a prediction can be derived by means of locally weighted linear regression. In this case, a linear regression model is fitted to the $\mathrm{k}$ nearest neighbors, and this model is used to make a prediction for the query instance, which sets the Prediction_Strategy parameter Local_Linear_Regression by selecting 'Loc_Lin_Reg' in the MOA framework

\subsection{Prediction Strategies}

In instance-based learning, a prediction for the query instance is obtained by combining, in one way or the other, the outputs of the neighbors of this instance in the training data. The type of aggregation depends on the type of problem to be solved. MOA offers four different prediction schemes, namely the Weighted_Mode for classification, the Weighted_Median for ordinal classification, and the Weighted_Mean and Local_Linear_Regression for regression problems. The present work uses only the prediction schemes.

\subsection{Adaptation Strategies}

Adapting to the size of the neighborhood effectively contributes for the performance of an IBLS. Two different adaption strategies used in the evaluation are explained as follows. The present work uses the first strategy:

\subsubsection{Adapting_ $k$}

In this case, the size of the neighborhood is controlled by the number of neighbors, $\mathrm{k}$, and the IBLS algorithm adapts this value by continuously checking whether it appears beneficial to increase or decrease the current value by 1 for evaluation. This adaptation method is enabled in the GUI of MOA configuration model the by setting the parameter to adapt_K.

\subsubsection{Adapting the Kernel Width}

In this case, the size of the neighborhood is controlled the weighting function or corresponding kernel width. The algorithm will then check whether variation in the value of kernel width sigma by a certain percentage appears to be beneficial for the evaluation. This is activated by enabling the option adapt_sigma in the GUI of MOA configuration.

\subsection{Weighting Methods}

Five different Weighting methods are proposed for the influence of the k-neighbors of the query instances. They are explained in brief as follows.

1. equal weighting method: All neighbors are given equal weight, namely $1 / \mathrm{k}$.

2. inverse Distance weighting method: The weight of an instance is proportional to $1 / \mathrm{d}$, where ' $\mathrm{d}$ ' is its distance from the query.

3. linear weighting method: The weight function is a linearly decreasing function of the distance $d$. The slope is determined such that the neighbor with the highest distance has a small weight of 0.001 , while an instance with distance 0 would have a weight of 1 . 
4. gaussian Kernel weighting method: Neighbors are weighted by centering a Gaussian kernel at the query instance.

5. exponential Kernel weighting method: Neighbors are weighted by centering an exponential kernel at the query instance.

\subsection{Selecting the Instance Width}

Two important parameters used in the GUI of MOA for selecting the instance width are initial_width and max_instance_base_size. Basically, the first parameter initial_width defines the size of the initial set of instances on which IBLS produces the initial model. After checking the number of instances, the algorithm switches to its incremental mode of learning using a batch wise learning mode. Normally the default value for increment is 1000 instances. Second important parameter is max_instance base_size which upper-bounds the size of the training set which uses the default value as 5000 instances.

\section{Experiments and Results}

Massive online analysis is the frame work used for mining data streams. The IBLS algorithm is implemented in MOA. IBLS is used as an extension to MOA. Configuration of the task launcher is configured for all the eight different data stream generators of MOA. (LED, HYPERPLANE, RANDOMRBF, WAVEFORM, AGRAWAL, SEA, STAGGER, RANDOMTREE) using both Basic Regression Performance Evaluator (BRPE) and Windows Regression Performance Evaluator(WRPE). The prediction strategies used are Local_Linear_Regression and Weighted_Mean_ Regression. In the regression case, the IBLS are used in four different settings (while the rest of the parameters were again set to their default values).

R1: weighted mean, equal weighting of neighbors, adaptation of neighborhood size $\mathrm{k}$.

R2: weighted mean, weighting with exponential kernel, adaptation of kernel width.

R3: local linear regression, equal weighting of neighbors, adaptation of neighborhood size $\mathrm{k}$.

R4: local linear regression, weighting with exponential kernel, adaptation of kernel width.

One sample setting for hyper plane data stream generator is shown in figure 1. From the above four settings it is R4. In addition to options of R1, BRPE is used. For the rest of the data stream generators the same type of settings are followed. The result window is shown in figure 2 and the results are tabulated in table1.

The performance of IBLstreams learner on eight data stream generators in MOA framework is predicted in table 1 . The experiment constitutes the evaluation of Mean Absolute Error (MAE) and Root mean square error (RMSE).The key features observed from the above table are presented below:

For any stream generator the prediction strategies $\mathrm{w}_{-}$ mean_reg and loc_lin_reg and they happen to be same.

For each prediction stretegy the performance evaluators are Basic Regression Performance Evaluator (BRPE) and Windows Regression Performance Evaluator(WRPE) and they happen to be same.

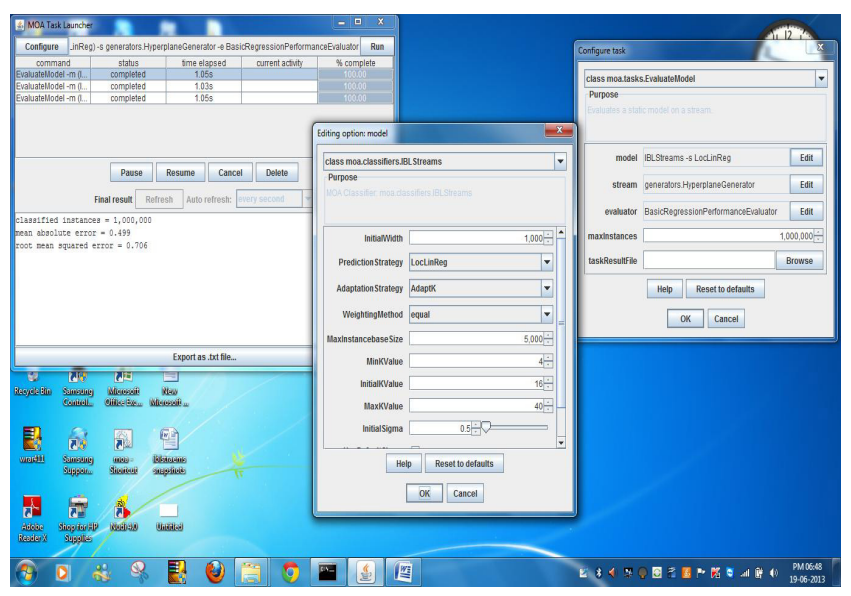

Figure 1. GUI for for Hyperplane Generator with R1 settings.

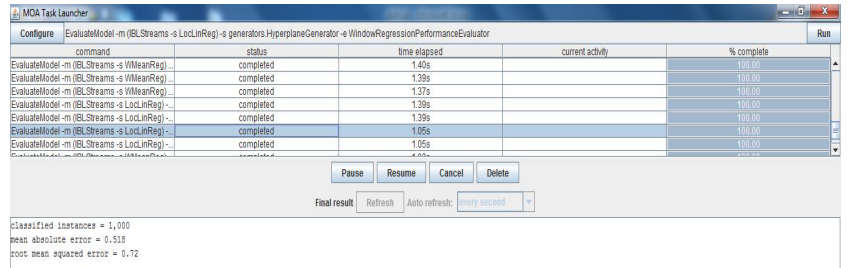

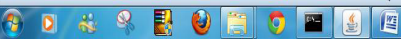

Figure 2. Result window for Hyperplane Generator with R1 settings. 
Table 1. Tabulation of Results for all data streams

\begin{tabular}{|c|c|c|c|c|}
\hline $\begin{array}{l}\text { DATA } \\
\text { STREAMS }\end{array}$ & $\begin{array}{c}\text { PREDICTION } \\
\text { STRATEGY }\end{array}$ & EVALUATOR & $\begin{array}{c}\text { MEAN } \\
\text { ABSOLUTE } \\
\text { ERROR (MAE) }\end{array}$ & $\begin{array}{c}\text { ROOT MEAN } \\
\text { SQUARE ERROR } \\
\text { (RMSE) }\end{array}$ \\
\hline \multirow{4}{*}{$\begin{array}{l}\text { RANDOM RBF } \\
\text { GENERATOR }\end{array}$} & \multirow[t]{2}{*}{ W_MEAN_REG } & BRPE & 0.502 & 0.708 \\
\hline & & WRPE & 0.505 & 0.711 \\
\hline & \multirow[t]{2}{*}{ LOC_LIN_REG } & BRPE & 0.502 & 0.708 \\
\hline & & WRPE & 0.505 & 0.711 \\
\hline \multirow{4}{*}{$\begin{array}{l}\text { RANDOM } \\
\text { TREE } \\
\text { GENERATOR }\end{array}$} & \multirow[t]{2}{*}{ W_MEAN_REG } & BRPE & 0.422 & 0.649 \\
\hline & & WRPE & 0.428 & 0.654 \\
\hline & \multirow[t]{2}{*}{ LOC_LIN_REG } & BRPE & 0.422 & 0.649 \\
\hline & & WRPE & 0.428 & 0.654 \\
\hline \multirow{4}{*}{$\begin{array}{l}\text { WAVE FORM } \\
\text { GENERATOR }\end{array}$} & \multirow[t]{2}{*}{ W_MEAN_REG } & BRPE & 0.667 & 0.819 \\
\hline & & WRPE & 0.674 & 0.821 \\
\hline & \multirow[t]{2}{*}{ LOC_LIN_REG } & BRPE & 0.667 & 0.819 \\
\hline & & WRPE & 0.674 & 0.821 \\
\hline \multirow{4}{*}{$\begin{array}{l}\text { AGARWAL } \\
\text { GENERATOR }\end{array}$} & \multirow[t]{2}{*}{ W_MEAN_REG } & BRPE & 0.672 & 0.82 \\
\hline & & WRPE & 0.681 & 0.825 \\
\hline & \multirow[t]{2}{*}{ LOC_LIN_REG } & BRPE & 0.672 & 0.82 \\
\hline & & WRPE & 0.681 & 0.825 \\
\hline \multirow{4}{*}{$\begin{array}{l}\text { SEA } \\
\text { GENERATOR }\end{array}$} & \multirow[t]{2}{*}{ W_MEAN_REG } & BRPE & 0.672 & 0.82 \\
\hline & & WRPE & 0.681 & 0.825 \\
\hline & \multirow[t]{2}{*}{ LOC_LIN_REG } & BRPE & 0.672 & 0.82 \\
\hline & & WRPE & 0.681 & 0.825 \\
\hline \multirow{4}{*}{$\begin{array}{l}\text { STAGGER } \\
\text { GENERATOR }\end{array}$} & \multirow[t]{2}{*}{ W_MEAN_REG } & BRPE & 0.112 & 0.334 \\
\hline & & WRPE & 0.103 & 0.321 \\
\hline & \multirow[t]{2}{*}{ LOC_LIN_REG } & BRPE & 0.112 & 0.334 \\
\hline & & WRPE & 0.103 & 0.321 \\
\hline \multirow{4}{*}{$\begin{array}{l}\text { LED } \\
\text { GENERATOR }\end{array}$} & \multirow[t]{2}{*}{ W_MEAN_REG } & BRPE & 3.702 & 4.53 \\
\hline & & WRPE & 3.524 & 4.376 \\
\hline & \multirow[t]{2}{*}{ LOC_LIN_REG } & BRPE & 3.702 & 4.53 \\
\hline & & WRPE & 3.524 & 4.376 \\
\hline \multirow{4}{*}{$\begin{array}{l}\text { HYPER PLANE } \\
\text { GENERATOR }\end{array}$} & \multirow[t]{2}{*}{ W_MEAN_REG } & BRPE & 0.499 & 0.706 \\
\hline & & WRPE & 0.518 & 0.72 \\
\hline & \multirow[t]{2}{*}{ LOC_LIN_REG } & BRPE & 0.499 & 0.706 \\
\hline & & WRPE & 0.518 & 0.72 \\
\hline
\end{tabular}

Eventually the values of MAE and RMSE are different for different data stream generators and minimum value is observed for stagger generator. i.e. for the case of BRPE and prediction stretegies w_mean_reg and loc_lin_reg, MAE $=0.112$, RMSE $=0.334$ and for the case of WRPE and prediction stretegies w_mean_reg and loc_ lin_reg, $\mathrm{MAE}=0.103, \mathrm{RMSE}=0.321$. This suggests that the performance of IBLstreams is excellent in the case of STAGGER generator

A care full survey of the litertaure ( our papers) reveals that the regression analysis could be performed not only on small data sets but also on data streams as in the present case but the method of analysis will be different in the two cases. In the case of small data set the regression 
models are linear, multiple and polynomial regression, while in the case of data streams the entire analysis is performed under the Massive Online Analysis framework by taking the two evaluation parameters basic regression performance evaluator and windows regression performance evaluator. This finding is first of its kind in literature and is quite interesting.

Based on table 1, the results are presented graphically in Figures 3, 4, 5 and 6, for all the setting options R1, R2, $\mathrm{R} 3$ and $\mathrm{R} 4$ respectively and are self explanatory.

\section{Conclusions}

Regression analysis is a part of machine learning process, widely used for prediction, when numerical values are involved.

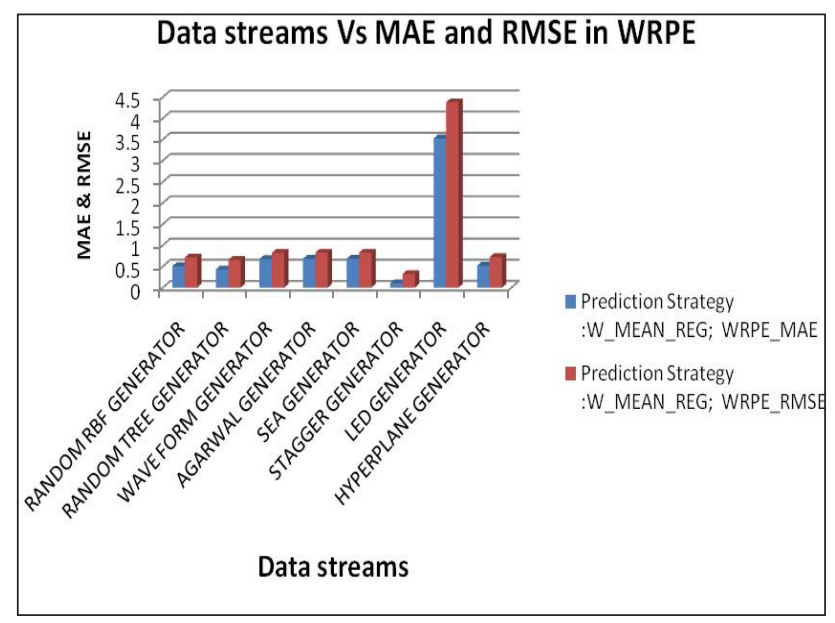

Figure 3. Graph of MAE and RMSE for Prediction Strategy = W_MEAN_REG, Performance Evaluator $=$ BRPE.

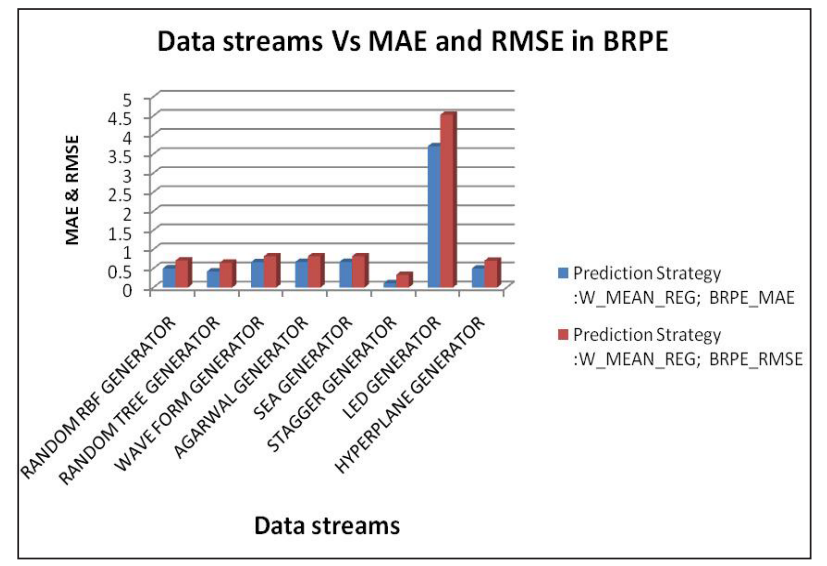

Figure 4. Graph of MAE and RMSE for Prediction Strategy = W_MEAN_REG, Performance Evaluator $=$ WRPE.
Regression analysis plays a very important role in modeling and analyzing several variables, when the focus is on the relationship between a dependent variable and one or more independent variables.

Traditional DM techniques are not suitable for mining data streams because of their ubiquitous nature.

Sophisticated techniques are required to mine data streams, Massive Online Analysis is one such frame work used for data stream mining.

Basic data mining techniques, classification, clustering and association rule mining can be performed on data streams using MOA framework.

The performance of IBLstreams learner on eight data stream generators in MOA framework is predicted.

The experiment constitutes the evaluation of Mean Absolute Error (MAE) and Root mean square error (RMSE).

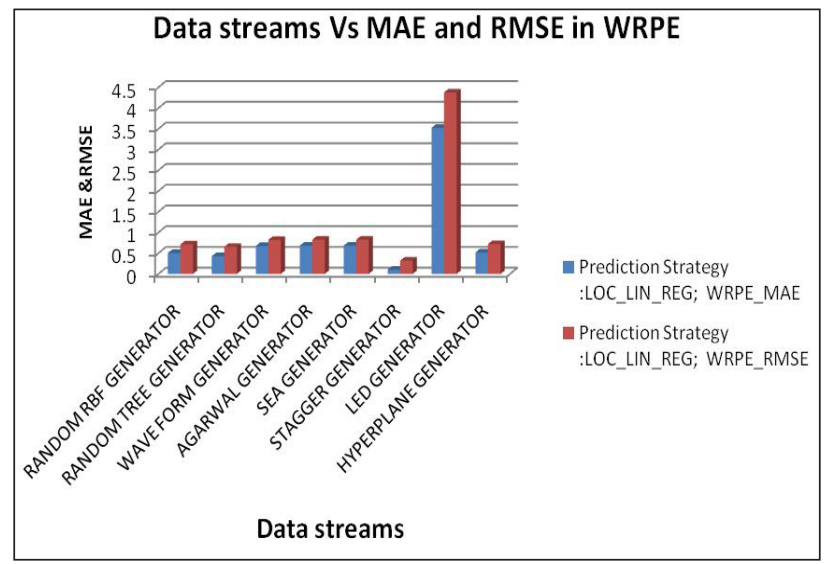

Figure 5. Graph of MAE and RMSE for Prediction Strategy =LOC_IN_REG, Performance Evaluator $=$ WRPE.

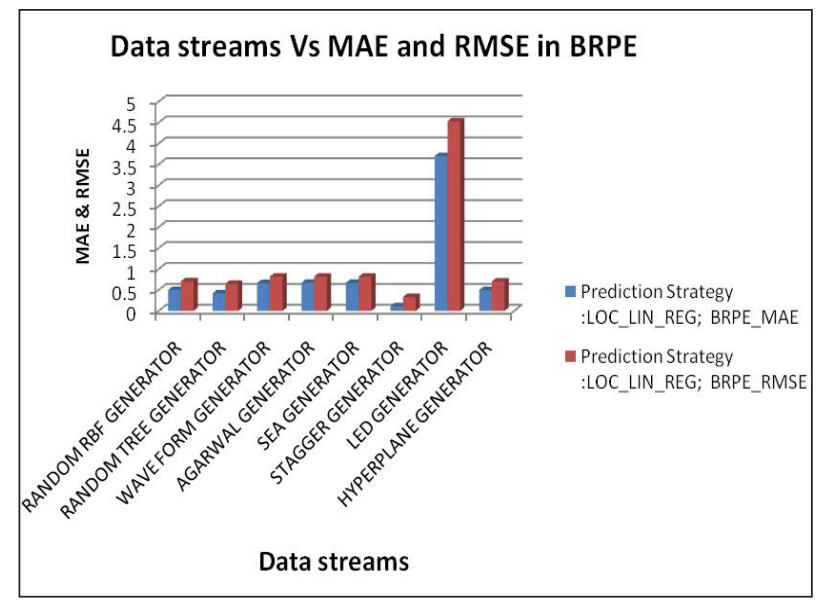

Figure 6. Graph of MAE and RMSE for Prediction Strategy =LOC_IN_REG, Performance Evaluator $=$ BRPE. 
The key features observed from the above investigation for any stream generator the prediction strategies are w_mean_reg and loc_lin_reg and evalutors are BRPE and WRPE.

The performance of IBLStreams is excellent in the case of STAGGER generator.

Finally it is concluded that regression analysis could be performed not only on small data sets but also on data streams as in the present case but the method of analysis will be different in the two cases. In the case of small data set the regression models are linear, multiple and polynomial regression, while in the case of data streams the entire analysis is performed under the Massive Online Analysis framework by taking the two evaluation parameters basic regression performance evaluator and windows regression performance evaluator.

This finding is first of its kind in literature and is quite interesting.

\section{Acknowledgement}

One of the authors Mrs. Malini M. Patil acknowledges JSSMVP's, J.S.S Academy of Technical education, Bangalore, Karnataka and Bharathiyaar University, Coimbatore, Tamilnadu, India for providing the facilities for carrying out the research work. The authors also would like to acknowledge Dr. Albert Bifet and Dr.Ammar Shakeer for their valuable suggestions in carrying out the present work.

\section{References}

1. Han J, Kamber M. Data mining: concepts and techniques. San Francisco, CA: Morgon Kaufmann Publishers; 2007.

2. Gaber MM, Zaslavsky A, Krishnamurthy S. Data streams: models and methods. 2007; 31:39-59.

3. Gaber MM, Gama J. State of art in data streams mining. ECML.; 2007.

4. Ikonomovska SL, Gjorgjevik D. A survey of data stream mining; 2007.
5. Srimani PK, Patil MM. Linear regression model for Edu_ data, International Journal of Conceptions on Electrical and Electronics Engineering. 2013; 1(1):45-49.

6. Srimani PK, Patil MM. Regression modelling on Edu_data in TES. International Journal of Advanced Scientfic and Technical Research. 2013; 1(3):320-36.

7. Srimani PK, Patil MM. Regression modelling on Edu_data in TES: a linear approach. ICT and Critical Infrastructure Proceedings of the 48th Annual Convention of Computer Society of India - Volume II, Advances in Intelligent Systems and Computing; 2014. p. 785-93.

8. PASW ${ }^{\circledR}$ Statistics 18. Brief Guide SPSS Inc; 2007.

9. Aggarwal C. Data streams: models and algorithms. Springer; 2007.

10. Leung Y, Ma J-H, Zhang W-X. A new method for mining regression classes in large data sets. IEEE Transactions on Pattern Analysis and Machine Intelligence. 2001; 23(1): 5-17.

11. Fan T-H, Cheng K-F. Tests and variables selection on regression analysis for massive datasets. Proceedings of the Second Workshop on Knowledge Economy and Electronic Commerce; 2004. p. 229-38.

12. Shaker A, Hüllermeier E. IBL Streams: A system for instance-based classification and regression on data streams. Evolving Systems. 2012; 3(4):235-49.

13. Lughofer E. FLEXFIS: A robust incremental learning approach for evolving Takagi-Sugeno fuzzy models. IEEE Transactions on Fuzzy Systems. 2008; 16(6):1393-410.

14. Bifet A, Holmes G, Pfahringer B, Gavald’a R. Improving adaptive bagging methods for evolving data streams. Lecture Notes in Computer Science. 2009; 23-37.

15. Bifet A, Holmes G, Pfahringer B, Kirkby R, Gavald a R. New ensemble methods for evolving data streams. KDD'09. ACM. 2009; 139-14.

16. Bifet A, Kirkby R. Data stream mining: A practical approach. Technicalreport.Newzeland:TheUniversityofWaikato;2009.

17. Bifet A, Holmes G, Pfahringer B, Kirkby R, Gavalda R. 'NewEnsemble methods for evolving data streams. 15th ACM International Conference on Knowledge Discovery and Data Mining; 2009.

18. Bifet A, Holmes G, Kirby R, Pfahringer B. MOA: Massive online analysis'. J Mach Learn Res. 2011; p. 1601-4. 